\title{
A defined, feeder-independent medium for human embryonic stem cell culture
}

\author{
Chris Lannon ${ }^{1}$, Jennifer Moody ${ }^{1}$, Debbie King ${ }^{1}$, Terry Thomas ${ }^{1}$, Allen Eaves ${ }^{2}$, Cindy Miller ${ }^{1}$ \\ ${ }^{I}$ Stem Cell Technologies Inc., Vancouver, BC, Canada; ${ }^{2}$ Terry Fox Laboratory, British Columbia Cancer Research Centre, \\ Vancouver, BC, Canada
}

Human embryonic stem cells (hESC) are derived from the epiblast tissue of the inner cell mass and are capable of giving rise to cells from all three germ layers - ectoderm, mesoderm, and endoderm. Although the basic techniques to create and culture hESC are well established, limitations remain in many of the procedures used for their culture. In particular, most existing hESC lines have been cultured with components derived from animal serum and using mouse embryonic fibroblast feeder layers. However, the use of serum or feeder layers induces variability in these culture systems, and limits their application in the clinical setting. Accordingly, there is considerable interest in the development of serum-free and feeder-independent hESC culture systems. mTeSR ${ }^{\text {TM}} 1$ is a fully-defined, serum-free medium for the derivation and maintenance of hESCs cultured on BD Matrigel ${ }^{\mathrm{TM}}$ hESC-qualified Matrix(Ludwig et al. Nat Methods 3(8):637-46). mTeSR ${ }^{\mathrm{TM} 1}$ has been demonstrated to support the undifferentiated culture of multiple hESC lines derived by independent sources, with examples including H1, H9 CA1, and SA121. Cells grown for multiple passages in mTeSR ${ }^{\text {TM}} 1$ maintain characteristic undifferentiated colony morphology, and FACS analysis confirms high expression of pluripotency-associated markers (Oct4, SSEA-3, SSEA4, TRA-1-60, and TRA-1-81), and the absence of expression of the differentiation marker SSEA-1. RT-PCR analysis of cells cultured in $\mathrm{mTeSR}^{\mathrm{TM}} 1$ also validate the continued expression of Oct 4 and Nanog and the lack of expression of multiple differentiation markers. Furthermore, hESC cultures maintained in $\mathrm{mTeSR}^{\mathrm{TM}} 1$ retain a high proliferative capacity and normal karyotype after extended culture. Finally, the teratoma formation assay verified the preserved potential of these cells to subsequently differentiate into tissues derived from all three germ layers. Together these results establish $\mathrm{mTeSR}^{\mathrm{TM}} 1$ as an optimal, standardized medium for researchers culturing hESC.

Keywords: human embryonic stem cells, defined media, feeder-free

Cell Research (2008) 18:s34. doi: 10.1038/cr.2008.124; published online 4 August 2008

Correspondence: Myriam Hofmeister

Email: myriam@stemcell.com 